\title{
A Note on Local Regularity of Axisymmetric Solutions to the Navier-Stokes Equations
}

\author{
G. Seregin(D) \\ Communicated by G. P. Galdi
}

\begin{abstract}
In the paper, a new slightly supercritical condition, providing local regularity of axially symmetric solutions to the non-stationary 3D Navier-Stokes equations, is discussed. It generalises almost all known results in the local regularity theory of weak axisymmetric solutions.
\end{abstract}

Keywords. Navier-Stokes equations, Axisymmetric solutions, Local regularity.

\section{Introduction}

In the submitted note, we study potential singularities of axially symmetric flows of viscous incompressible fluids. It has been shown in the paper [17] that an axisymmetric solution to the three-dimensional nonstationary Navier-Stokes equations with a bounded scale-invariant energy quantity is actually smooth, i.e., axially symmetric solutions do no exhibit Type I blowups (see the paper [17] for more definitions and explanations).

Let us consider the 3D non-stationary Navier-Stokes system

$$
\partial_{t} v+v \cdot \nabla v-\Delta v=-\nabla q, \quad \operatorname{div} v=0
$$

in the space-time parabolic cylinder $Q=\mathcal{C} \times]-1,0\left[\right.$, where $\mathcal{C}=\left\{x=\left(x_{1}, x_{2}, x_{3}\right): x_{1}^{2}+x_{2}^{2}<1,-1<\right.$ $\left.x_{3}<1\right\}$ is a usual right circle cylinder in $\mathbb{R}^{3}$. In what follows, it is supposed that the pair $v$ and $q$ is the so-called suitable weak solution to the Navier-Stokes equations in $Q$. We recall a definition of such solutions.

Definition 1.1. Let $\omega \subset \mathbb{R}^{3}$ and $T_{2}>T_{1}$. The pair $w$ and $r$ is a suitable weak solution to the Navier-Stokes system in $\left.Q_{*}=\omega \times\right] T_{1}, T_{2}[$ if:

1. $w \in L_{2, \infty}\left(Q_{*}\right), \nabla w \in L_{2}\left(Q_{*}\right), r \in L_{\frac{3}{2}}\left(Q_{*}\right)$;

2. $w$ and $r$ satisfy the Navier-Stokes equations in $Q_{*}$ in the sense of distributions;

3. for a.a. $t \in\left[T_{1}, T_{2}\right]$, the local energy inequality

$$
\begin{gathered}
\int_{\omega} \varphi(x, t)|w(x, t)|^{2} d x+2 \int_{T_{1}}^{t} \int_{\omega} \varphi|\nabla w|^{2} d x d t^{\prime} \leq \int_{T_{1}}^{t} \int_{\omega}\left[|w|^{2}\left(\partial_{t} \varphi+\Delta \varphi\right)+\right. \\
\left.+w \cdot \nabla \varphi\left(|w|^{2}+2 r\right)\right] d x d t^{\prime}
\end{gathered}
$$

holds for all non-negative $\varphi \in C_{0}^{1}(\omega \times] T_{1}, T_{2}+\left(T_{2}-T_{1}\right) / 2[)$.

To the memory of Olga Alexandrovna Ladyzhenskaya. 
Our standing assumption is that a suitable weak solution $v$ and $q$ to the Navier-Stokes equations in $Q=\mathcal{C} \times]-1,0$ [ is axially symmetric with respect to $x_{3}$-axis. It means the following: if we introduce the corresponding cylindrical coordinates $\left(r, \theta, x_{3}\right)$ and use the corresponding representation $v=v_{r} e_{r}+v_{\theta} e_{\theta}+$ $v_{3} e_{3}$, then $v_{r, \theta}=v_{\theta, \theta}=v_{3, \theta}=q_{, \theta}=0$. Here, the comma in lower indices means the partial derivative in the indicated spatial direction.

With the regards to the state of arts in the regularity theory of axially symmetric solutions to the Navier-Stokes equations, we could refer to the previous papers [17] and [18] of the author and especially to references therein. For example, one could mention the following very interesting papers: [6], [23], [10], [14], [16], [2], [21], [4], [19], [8], [15], [9], [3], [22], and [25].

In a sense, our note is a continuation of author's paper [18], which, in turn, has been inspired by paper [15] of X. Pan, where the regularity of solutions has been proved under a slightly supercritical assumption. The aims of the present paper are to consider a local setting and a different supercritical assumption.

In order to describe our supercritical assumption, additional notation is needed. Given $x=\left(x_{1}, x_{2}, x_{3}\right) \in$ $\mathbb{R}^{3}$, denote $x^{\prime}=\left(x_{1}, x_{2}, 0\right)$. Next, different types of spatial cylinders will be denoted as $\mathcal{C}(r)=\{x$ : $\left.\left.\left|x^{\prime}\right|<r,\left|x_{3}\right|<r\right\}, \mathcal{C}\left(x_{0}, r\right)=\mathcal{C}(r)+x_{0}, Q^{\lambda, \mu}(r)=\mathcal{C}(\lambda r) \times\right]-\mu r^{2}, 0\left[, Q^{1,1}(r)=Q(r), Q^{\lambda, \mu}\left(z_{0}, r\right)=\right.$ $\left.\mathcal{C}\left(x_{0}, \lambda r\right) \times\right] t_{0}-\mu r^{2}, t_{0}[$. And, finally, let

$$
f(R):=\frac{1}{\sqrt{R}}\left(\int_{-R^{2}}^{0}\left(\int_{\mathcal{C}(R)}|\bar{v}|^{3} d x\right)^{\frac{4}{3}} d t\right)^{\frac{3}{4}}
$$

and

$$
M(R):=\frac{1}{\sqrt{R}}\left(\int_{Q(R)}|\bar{v}|^{\frac{10}{3}} d z\right)^{\frac{3}{10}}
$$

for any $0<R \leq 1$, with $\bar{v}=v_{r} e_{r}+v_{3} e_{3}$, and assume that:

$$
f(R)+M(R) \leq g(R):=c_{*} \ln ^{\alpha} \ln ^{\frac{1}{2}}(1 / R)
$$

for all $0<R \leq 2 / 3$, where $c_{*}$ and $\alpha$ are positive constants and $\alpha$ obeys the condition:

$$
0<\alpha \leq \frac{1}{224} \text {. }
$$

In the paper [18], the following completely local result has been stated.

Theorem 1.2. Assume that a pair $v$ and $q$ is an axially symmetric suitable weak solution to the NavierStokes equations in $Q$ and let conditions (1.2) and (1.3) hold. Then the origin $z=0$ is a regular point of $v$.

Unfortunately, the above theorem has not been proven in [18]. Instead, a global version of it has been stated and demonstrated there. However, a big step toward a proof of Theorem 1.2 has been made in that paper [18]. It is a careful analysis of the scalar equation

$$
\partial_{t} \sigma+\left(v+2 \frac{x^{\prime}}{\left|x^{\prime}\right|^{2}}\right) \cdot \nabla \sigma-\Delta \sigma=0,
$$

being held in domain $Q \backslash\left(\left\{x^{\prime}=0\right\} \times\right]-1,0[)$. Here, $\sigma:=\varrho v_{\varphi}=v_{2} x_{1}-v_{1} x_{2}$.

Let us recall known differentiability properties of $\sigma$. Most of them follow from the partial regularity theory developed by Caffarelli-Kohn-Nirenberg in their famous paper [1]. As to further discussions and improvements, see also papers [12] and [7] and of course references therein.

Indeed, since $v$ and $q$ are an axially symmetric suitable weak solution in $Q$, there exists a closed subset $S^{\sigma}$ of $Q$, whose 1D-parabolic measure in $\mathbb{R}^{3} \times \mathbb{R}$ is equal to zero and $x^{\prime}=0$ for any $z=(x, t) \in S^{\sigma}$, such that any spatial derivative of $v$ with respect to Cartesian coordinates (and thus of $\sigma$ ) is Hölder continuous in $Q \backslash S^{\sigma}$ in space-time. 
Next,

$$
\sigma \in W_{p}^{2,1}(P(\delta, R ; R) \times]-R^{2}, 0[)
$$

for any $0<\delta<R<1$ and for any finite exponent $p \geq 2$.

One can show also that $\sigma \in L_{\infty}(Q(R))$ for any $0<R<1$, see, for example, papers [19] and [17].

What actually has been proved in paper [18], see also the last section of the present paper, is as follows:

Proposition 1.3. Assume that all assumptions of Theorem 1.2 hold. Let $\sigma=\varrho v_{\varphi}$, then

$$
\operatorname{OSc}_{z \in Q(r)} \leq e^{-c\left[\ln \frac{1}{4}(1 /(2 r))-\ln \frac{1}{4}(1 /(2 R))\right]} \operatorname{OSc}_{z \in Q(2 R)} \sigma(z)
$$

for all $0<r<R \leq R_{*}\left(c_{*}, \alpha\right) \leq 1 / 6$, where $c$ is a positive absolute constant.

Here, $\operatorname{osc}_{z \in Q(r)} \sigma(z)=M_{r}-m_{r}$ and

$$
M_{r}=\sup _{z \in Q(r)} \sigma(z), \quad m_{r}=\inf _{z \in Q(r)} \sigma(z) .
$$

Now, the main task of the note is to deduce Theorem 1.2 from Proposition 1.3.

Let us describe briefly the most important counter-parts of our arguments. The first one is a choice of a cut-off function that takes into account the well known partial regularity of axisymmetric flows. The second important point is a local regularity properties of solution $\hat{v}$ to the following elliptic system:

$$
\operatorname{div} \bar{v}=0, \quad \operatorname{curl} \bar{v}=\omega_{\theta} e_{\theta}
$$

in $\mathcal{C}$, where $\omega_{\theta}=v_{r, 3}-v_{3, r}$ is the corresponding component of the vorticity field $\omega=\operatorname{curl} v$, see Lemma 2.3 of Section 2. It should be mentioned that Lemma 2.3 of the present note is a local version of the corresponding global statement, see [13], Proposition 2.5 therein, or [3], Lemma 2.3 therein.

In Section 3, we show how Pan type results on supercritical regularity, see Pan's paper [15], can be deduced from Theorem 1.2 of the present note. To be a bit more precise, let us replace the main supercritical condition (1.2) with the other one, where, for simplicity, it is assumed that:

$$
\left|v\left(r, x_{3}, t\right)\right| \leq \frac{c}{r} \ln ^{\alpha} \ln \frac{1}{r}
$$

in $Q$, with a positive number $\alpha$.

Theorem 1.4. Assume that $v$ and $q$ are suitable weak solution to the Navier-Stokes equations in $Q$ and condition (1.7) is satisfied with $0<\alpha<4 / 7$. Then, condition (1.2) holds as well but with new exponent $35 \alpha / 6$ and a new constant $c$, depending on $\alpha$, and some energy quantities of $v$ and $q$.

As it follows from Theorem 1.2, supercritical condition (1.7) implies regularity of the velocity field $v$ at the origin $z=0$ for sufficiently small $\alpha$.

At the end of the section, we would like to comment our notation. All absolute constants are denoted by $c$, other constants are denoted by $C$ with indication of variables of which those constants may depend on. The norms of the Lebesgue space $L_{p}(\omega)$ are denoted by $\|\cdot\|_{p, \omega}$ and of the mixed Lebesgue space $L_{p, q}\left(Q_{T}\right)=L_{q}\left(0, T ; L_{p}(\omega)\right)$ are denoted by $\|\cdot\|_{p, q, Q_{T}}$, where $\left.Q_{T}=\omega \times\right] 0, T[$.

\section{Proof of Theorem 1.2}

Step 1. Construction of a cut-off function. The partial regularity theory for the Navier-Stokes equations implies that if singular points of an axisymmetric velocity field $v$ exist, they must belong to the axis of symmetry, which is $x_{3}$-axis in our case. Since the $1 \mathrm{D}$ parabolic Hausdorff measure of the set of singular points is equal to zero, there exist at least two regular points $z_{1}=\left(x^{\prime}, x_{3}, t\right)=\left(0, h_{1}, 0\right)$ and $z_{2}=$ $\left(0,-h_{2}, 0\right)$ of $v$ such that $0<h_{1}, h_{2}<1$. According to the properties of regular points, there are cylinders $Q_{1}=Q\left(z_{1}, \delta\right)$ and $Q_{2}=Q\left(z_{2}, \delta\right)$ with radius $\delta>0$ such that $v \in C\left(\left[-\delta^{2}, 0\right] ; C^{3}\left(\overline{\mathcal{C}}\left(\left(0, h_{1}\right), \delta\right)\right)\right) \cap$ 
$C\left(\left[-\delta^{2}, 0\right] ; C^{3}\left(\overline{\mathcal{C}}\left(\left(0,-h_{2}\right), \delta\right)\right)\right)$ at least. Moreover, one can find $\left.t_{0} \in\right]-\delta^{2}, 0[$ such that there is no singular point in the set $\left.\overline{\mathcal{C}}\left(r_{0}\right) \times\left[t_{0}, t_{0}+\delta_{0}^{2}\right] \subset\right]-t_{0}, 0\left[\right.$ with $0<r_{0}<1$ and $\delta_{0}>0$. It is also possible to pick up $r_{0}$ close to one so that $r_{0} \geq \max \left\{h_{1}-\delta, h_{2}-\delta\right\}$.

Without loss of generality (just for simplicity of calculations), we may assume that $h_{1}=h_{2}=3 / 4$, $\delta=1 / 8$, and $t_{0}=-1 / 32, t_{0}+\delta_{0}=-1 / 64, r_{0}=4 / 5$. Then we are going to use a smooth cut-off function $\eta\left(r, x_{3}, t\right)=\varphi(r) \psi\left(x_{3}\right) \xi(t)$, where $\varphi, \psi$, and $\xi$ are smooth functions having the following properties:

- all of them take the values in $[0,1]$;

- $\varphi(r)=1$ if $0 \leq r<1 / 2 ; \varphi(r)=0$ if $r \geq 5 / 6$

- $\psi\left(x_{3}\right)=1$ if $0 \leq x_{3}<1 / 2 ; \psi\left(x_{3}\right)=0$ if $x_{3} \geq 5 / 6$

- $\xi(t)=1$ if $t \geq-1 / 64 ; \xi(t)=0$ if $t \leq-1 / 32$.

Without loss of generality, we may assume also that

$$
\left|\sigma\left(r, x_{3}, t\right)\right|=r\left|v_{\theta}\left(r, x_{3}, t\right)\right| \leq C_{1} e^{-c \ln \frac{1}{4}(1 / r)}
$$

in $\mathcal{C}$. It is easy to see that $(2.1)$ implies

$$
\left|\sigma\left(r, x_{3}, t\right)\right|=r\left|v_{\theta}\left(r, x_{3}, t\right)\right| \leq \frac{c C_{1}}{\ln ^{3}(e / r)}
$$

for the same $x$ and $t$.

Step 2. Auxiliary Lemma.

Lemma 2.1. Let $\bar{v}=v_{r} e_{r}+v_{3} e_{3}$, where $v$ is our suitable weak solution, and let $\Gamma=\omega_{\theta} / r$. Then, for all $t \in]-1,0[$, the following estimates are valid:

$$
\left\|\nabla\left(\eta^{3} v_{r} / r\right)(\cdot, t)\right\|_{2, \mathcal{C}} \leq c\left\|\eta^{3} \Gamma(\cdot, t)\right\|_{2, \mathcal{C}}+C(v, \eta)
$$

and

$$
\left\|\bar{\nabla}^{2}\left(\eta^{3} v_{r} / r\right)(\cdot, t)\right\|_{2, \mathcal{C}} \leq c\left\|\eta^{3} \Gamma_{, 3}(\cdot, t)\right\|_{2, \mathcal{C}}+C(v, \eta) .
$$

Here, $\left|\bar{\nabla}^{2} g\right|^{2}=\left|g_{, r r}\right|^{2}+2\left|g_{, r 3}\right|^{2}+\left|g_{, 33}\right|^{2}$.

Proof. In order to prove the lemma, let us make use of elliptic system (1.6), where time $t \in]-1,0[$ is considered as a parameter. Introducing $\zeta=\eta^{3}$, derive from (1.6) the non-homogeneous system:

$$
\operatorname{div}(\zeta \bar{v})=\bar{v} \cdot \nabla \zeta, \quad \operatorname{curl}(\zeta \bar{v})=\zeta \omega_{\theta} e_{\theta}+\nabla \zeta \times \bar{v}
$$

in $\mathbb{R}^{3}$. In turn, from the above system, it follows that:

$$
-\Delta(\zeta \bar{v})=\operatorname{curl}\left(\zeta \omega_{\theta} e_{\theta}\right)-\nabla(\bar{v} \cdot \nabla \zeta)+\operatorname{curl}(\nabla \zeta \times \bar{v}) .
$$

Next, as a result of simple transformations, the following equation for $v_{r}$ can be deduced from (2.3):

$$
-\Delta\left(\zeta v_{r} e_{r}\right)=-\left(\left(\zeta \omega_{\theta}\right)_{, 3}+g\right) e_{r}-\left((\bar{v} \cdot \nabla \zeta)_{, 1},(\bar{v} \cdot \nabla \zeta)_{, 2}, 0\right),
$$

where $g=\left(\zeta_{, 3} v_{r}-\zeta_{, r} v_{3}\right)_{, 3}$. The latter equation can be solved in the whole space $\mathbb{R}^{3}$ so that

$$
\begin{gathered}
\frac{v_{r}}{r}=-\frac{1}{r} e_{r} \cdot \Delta^{-1}\left(\left(\zeta \omega_{\theta}\right)_{, 3} e_{r}\right)+\frac{1}{r} e_{r} \cdot \Delta^{-1}\left(G_{, 1}, G_{, 2}, 0\right) \\
-\frac{1}{r} e_{r} \cdot \Delta^{-1}\left((\bar{v} \cdot \nabla \zeta)_{, 1},(\bar{v} \cdot \nabla \zeta)_{, 2}, 0\right),
\end{gathered}
$$

where

$$
G\left(r, x_{3}, t\right)=\int_{r}^{\infty} g\left(\varrho, x_{3}, t\right) d \varrho
$$

and the operator $\Delta^{-1}$ is defined by Newton potential, i.e.,

$$
\Delta^{-1} h(x)=\frac{1}{4 \pi_{0}} \int_{\mathbb{R}^{3}} \frac{1}{|x-y|} h(y) d y
$$


for smooth compactly supported functions $h$. Here, $\pi_{0}=3,14 \ldots$

So,

$$
\frac{v_{r}}{r}=W+Z
$$

where

$$
W=-\frac{1}{r} e_{r} \cdot \Delta^{-1}\left(\left(\zeta \omega_{\theta}\right)_{, 3} e_{r}\right), \quad Z=\frac{1}{r} Z_{0, r}, \quad Z_{0}=\Delta^{-1}(G-\bar{v} \cdot \nabla \zeta) .
$$

Function $f=G-\bar{v} \cdot \nabla \zeta$ is axisymmetric, so does function $Z_{0}=\Delta^{-1} f$. Hence, according to Proposition 2.9 of paper [5], the following identity is valid:

$$
Z=\sin ^{2} \theta Z_{0,11}-2 \cos \theta \sin \theta Z_{0,12}+\cos ^{2} \theta Z_{0,22} .
$$

Next, exploiting the properties of singular integrals, one can derive two estimates:

$$
\|\nabla Z\|_{2, \mathbb{R}^{3}} \leq c\left\|\nabla_{x^{\prime}} f\right\|_{2, \mathbb{R}^{3}}
$$

and

$$
\left\|\bar{\nabla}^{2} Z\right\|_{2, \mathbb{R}^{3}} \leq c\left\|\nabla_{x^{\prime}}^{2} f\right\|_{2, \mathbb{R}^{3}} .
$$

Again, it is important to notice that $f\left(r, x_{3}, t\right) \neq 0$ only if $\left|\nabla \eta\left(r, x_{3}, t\right)\right|>0$. In the set $\operatorname{supp}|\nabla \eta|$, functions $v, \nabla v$, and $\nabla^{2} v$ are bounded in space-time. The most dangerous term on the right hand side of the latter inequalities are those where cut-off function $\eta$ does not contain derivatives in $r$. These terms contain $v_{3}, v_{r, 3}, v_{3, r}, v_{3, r r}, v_{3, r} / r, v_{r, 3 r}$, and $v_{r, 3} / r$. All of them are bounded by either $|v|$, or $|\nabla v|$, or $\left|\nabla^{2} v\right|$. So, $\left|\nabla Z_{0}(\cdot, t)\right|+\left|\bar{\nabla}^{2} Z_{0}(\cdot, t)\right| \leq C(v, \eta)$.

As to the function $W$, the global estimates have been already established in [3]. Here, they are:

$$
\|\nabla W\|_{2, \mathbb{R}^{3}} \leq c\|\zeta \Gamma\|_{2, \mathbb{R}^{3}}, \quad\left\|\bar{\nabla}^{2} W\right\|_{2, \mathbb{R}^{3}} \leq c\left\|(\zeta \Gamma)_{, 3}\right\|_{2, \mathbb{R}^{3}} .
$$

This completes the proof of the lemma.

Step 3. Local estimates of solutions. In this section, our goal is to make arguments of papers [24] and [3] completely local.

It is easy to verify that functions $\Phi=\omega_{r} / r=-v_{\theta, 3} / r$ and $\Gamma=\omega_{\theta} / r=\left(v_{r, 3}-v_{3, r}\right) / r$ satisfy the following equations:

$$
\begin{aligned}
& \partial_{t} \Phi+\left(v-\frac{2 x^{\prime}}{\left|x^{\prime}\right|^{2}}\right) \cdot \nabla \Phi-\Delta \Phi+\omega \cdot \nabla\left(\frac{v_{r}}{r}\right)=0, \\
& \partial_{t} \Gamma+\left(v-\frac{2 x^{\prime}}{\left|x^{\prime}\right|^{2}}\right) \cdot \nabla \Gamma-\Delta \Gamma+2 \frac{v_{\theta}}{r} \Phi=0 .
\end{aligned}
$$

Let us multiply the first equation by $\Phi \eta^{6}$ and the second equation by $\Gamma \eta^{6}$. After integration by parts, we find:

$$
\begin{aligned}
& \frac{1}{2} \partial_{t} \int_{\mathcal{C}}\left(\Phi \eta^{3}\right)^{2} d x+\int_{\mathcal{C}}\left(\eta^{3}|\nabla \Phi|\right)^{2} d x+\left.\pi_{0} \int_{-1}^{1}\left(\eta^{3} \Phi\right)^{2}\right|_{x^{\prime}=0} d x_{3} \\
& =\frac{1}{2} \int_{\mathcal{C}} \Phi^{2}\left(\partial_{t} \eta^{6}+\Delta \eta^{6}\right)+\int_{\mathcal{C}}\left(v-\frac{2 x^{\prime}}{\left|x^{\prime}\right|^{2}}\right) \cdot \nabla \eta^{6} \Phi^{2} d x \\
& \quad+\int_{\mathcal{C}}\left(v_{\theta}\left(\frac{v_{r}}{r}\right)_{, 3}\left(\Phi \eta^{6}\right)_{, r}-v_{\theta}\left(\frac{v_{r}}{r}\right)_{, r}\left(\Phi \eta^{6}\right)_{, 3}\right) d x=A_{1}+A_{2}+A_{3}
\end{aligned}
$$


and

$$
\begin{aligned}
\frac{1}{2} \partial_{t} \int_{\mathcal{C}}\left(\Gamma \eta^{3}\right)^{2} d x+\int_{\mathcal{C}}\left(\eta^{3}|\nabla \Gamma|\right)^{2} d x+\left.\pi_{0} \int_{-1}^{1}\left(\eta^{3} \Gamma\right)^{2}\right|_{x^{\prime}=0} d x_{3} \\
=\frac{1}{2} \int_{\mathcal{C}} \Gamma^{2}\left(\partial_{t} \eta^{6}+\Delta \eta^{6}\right)+\int_{\mathcal{C}}\left(v-\frac{2 x^{\prime}}{\left|x^{\prime}\right|^{2}}\right) \cdot \nabla \eta^{6} \Gamma^{2} d x \\
\quad-2 \int_{\mathcal{C}} \frac{v_{\theta}}{r} \Phi \eta^{6} \Gamma d x=B_{1}+B_{2}+B_{3} .
\end{aligned}
$$

Now, we wish to evaluate quantities $A_{i}$ and $B_{i}$, starting with $A_{1}$ and $B_{1}$ and letting $\Psi=\partial_{t} \eta^{6}+\Delta \eta^{6}$ to simplify our notation. Indeed, by the construction of our cut-off function $\eta$, the solution $v$ is smooth in the domain where $|\Psi|>0$. Now, it remains to use inequality $|\Gamma|^{2}+|\Phi|^{2} \leq|\nabla \omega|^{2}$ and boundedness of $|\nabla \omega|$ in the corresponding space-time domain. So, we have

$$
A_{1}+B_{1}=\int_{\mathcal{C}}\left(|\Phi|^{2}+|\Gamma|^{2}\right) \Psi d x \leq \int_{\mathcal{C}}|\nabla \omega|^{2}|\Psi| d x \leq C(v, \eta) .
$$

In order to estimate $A_{2}$ and $B_{2}$, boundedness of $v$ and its spatial derivatives over the support of $\nabla \eta$ and the obvious identity

$$
\frac{x^{\prime}}{\left|x^{\prime}\right|^{2}} \cdot \nabla \eta=\frac{1}{\left|x^{\prime}\right|}(\eta)_{, r} \leq C(\eta)
$$

are used. So, we have

$$
A_{2}+B_{2} \leq C(v, \eta) \int_{\frac{1}{2}<\left|x^{\prime}\right|<1} \eta^{5}\left(|\Gamma|^{2}+|\Phi|^{2}\right) d x .
$$

Since $|\Gamma|^{2}+|\Phi|^{2} \leq|\nabla \omega|^{2}$ and $|\nabla \omega|$ is bounded in $\left\{\frac{1}{2}<\left|x^{\prime}\right|<1\right\}$, the estimate

$$
A_{2}+B_{2} \leq C(v, \eta)
$$

is easily derived.

Our next goal is to find bounds for $A_{3}$ and $B_{3}$. To this end, let us fix a number $0<r_{1}<1 / 4$ and introduce domain $S_{1}=\left\{x \in \mathcal{C}:\left|x^{\prime}\right|<r_{1}\right\}$. Then,

$$
\begin{aligned}
B_{3}= & -2 \int_{S_{1}} \frac{v_{\theta}}{r}\left(\eta^{3} \Phi\right)\left(\eta^{3} \Gamma\right)+\frac{1}{r_{1}} C(v, \eta) \\
\leq & \frac{c C_{1}}{\ln \left(e / r_{1}\right)}\left(\int_{\mathcal{C}} \frac{1}{r^{2} \ln ^{2}(e / r)}\left|\eta^{3} \Gamma\right|^{2} d x\right)^{\frac{1}{2}} \\
& \left(\int_{\mathcal{C}} \frac{1}{r^{2} \ln ^{2}(e / r)}\left|\eta^{3} \Phi\right|^{2} d x\right)^{\frac{1}{2}}+\frac{1}{r_{1}} C(v, \eta) .
\end{aligned}
$$

In order to estimate the right hand side of the latter inequality, we are going to use a Leray type inequality in dimension two. For the reader convenience, let us state it for our particular case. The proof can be done with the help of integration by parts.

Lemma 2.2. For any function $f \in C_{0}^{1}(\mathcal{C})$, the following inequality is valid:

$$
\int_{\mathcal{C}} \frac{|f|^{2}}{\left|x^{\prime}\right|^{2} \ln ^{2}\left(e /\left|x^{\prime}\right|\right)} d x \leq 4 \int_{\mathcal{C}}\left|\nabla_{x^{\prime}} f\right|^{2} d x
$$


Applying Lemma 2.2, we find

$$
\begin{aligned}
B_{3} \leq & \frac{c C_{1}}{\ln \left(e / r_{1}\right)}\left\|\nabla_{x^{\prime}}\left(\eta^{3} \Gamma\right)\right\|_{2, \mathcal{C}}\left\|\nabla_{x^{\prime}}\left(\eta^{3} \Phi\right)\right\|_{2, \mathcal{C}}+\frac{1}{r_{1}} C(v, \eta) \\
\leq & \frac{c C_{1}}{\ln \left(e / r_{1}\right)}\left(\left\|\eta^{3} \nabla_{x^{\prime}} \Gamma\right\|_{2, \mathcal{C}}+\left\|\Gamma \nabla_{x^{\prime}} \eta^{3}\right\|_{2, \mathcal{C}}\right)\left(\left\|\eta^{3} \nabla_{x^{\prime}} \Phi\right\|_{2, \mathcal{C}}\right. \\
& \left.+\left\|\Phi \nabla_{x^{\prime}} \eta^{3}\right\|_{2, \mathcal{C}}\right)+\frac{1}{r_{1}} C(v, \eta) .
\end{aligned}
$$

Now, let us exploit one more time the fact that our solution $v$ has bounded spatial derivatives of any order in the support of $\nabla \eta$ and inequality $|\Gamma|^{2}+|\Phi|^{2}=\left(\left|\omega_{r}\right|^{2}+\left|\omega_{\theta}\right|^{2}\right) / r^{2} \leq|\nabla \omega|^{2}$. So,

$$
\begin{aligned}
B_{3} \leq & \frac{c C_{1}}{\ln \left(e / r_{1}\right)}\left\|\eta^{3} \nabla_{x^{\prime}} \Gamma\right\|_{2, \mathcal{C}}\left\|\eta^{3} \nabla_{x^{\prime}} \Phi\right\|_{2, \mathcal{C}} \\
& +C\left(v, \eta, r_{1}\right)\left(\left\|\eta^{3} \nabla_{x^{\prime}} \Gamma\right\|_{2, \mathcal{C}}+\left\|\eta^{3} \nabla_{x^{\prime}} \Phi\right\|_{2, \mathcal{C}}\right)+C\left(v, \eta, r_{1}\right) .
\end{aligned}
$$

As to the last quantity $A_{3}$, we find

$$
\begin{aligned}
A_{3}= & \int_{\mathcal{C}}\left(v_{\theta}\left(\frac{v_{r}}{r}\right)_{, 3} \eta^{3}\left(\eta^{3} \Phi\right)_{, r}-v_{\theta}\left(\frac{v_{r}}{r}\right)_{, r} \eta^{3}\left(\eta^{3} \Phi\right)_{, 3}\right) d x \\
& +\int_{\mathcal{C}}\left(v_{\theta}\left(\frac{v_{r}}{r}\right)_{, 3} \eta^{3} \Phi\left(\eta^{3}\right)_{, r}-v_{\theta}\left(\frac{v_{r}}{r}\right)_{, r} \eta^{3} \Phi\left(\eta^{3}\right)_{, 3}\right) d x=A_{31}+A_{32} .
\end{aligned}
$$

The first term on the right hand side can be transformed so that:

$$
\begin{aligned}
A_{31}= & \int_{\mathcal{C}}\left(v_{\theta}\left(\frac{\eta^{3} v_{r}}{r}\right)_{, 3}\left(\eta^{3} \Phi\right)_{, r}-v_{\theta}\left(\frac{\eta^{3} v_{r}}{r}\right)_{, r}\left(\eta^{3} \Phi\right)_{, 3}\right) d x \\
& -\int_{\mathcal{C}}\left(v_{\theta}\left(\frac{v_{r}}{r}\right)\left(\eta^{3}\right)_{, 3}\left(\eta^{3} \Phi\right)_{, r}-v_{\theta}\left(\frac{v_{r}}{r}\right)\left(\eta^{3}\right)_{, r}\left(\eta^{3} \Phi\right)_{, 3}\right) d x=A_{0}+A_{31}^{\prime} .
\end{aligned}
$$

Since $\left|v_{r} / r\right| \leq|\nabla v|$ and since the second integral is taken over support of $\nabla \eta$, we find

$$
\left.A_{31}^{\prime} \leq C(v, \eta)\left\|\nabla\left(\eta^{3} \Phi\right)\right\|_{2, \mathcal{C}} \leq C(v, \eta)\left(\| \eta^{3} \nabla \Phi\right) \|_{2, \mathcal{C}}+1\right) .
$$

To evaluate $A_{0}$, we may use Hölder inequality and the same trick as above. It gives us to the bound:

$$
\begin{aligned}
A_{0} & \leq c C_{1}^{2}\left(\int_{\mathbb{C}} \frac{1}{r^{2} \ln ^{6}\left(e /\left|x^{\prime}\right|\right)}\left(\left|\left(\frac{\eta^{3} v_{r}}{r}\right)_{, 3}\right|^{2}+\left|\left(\frac{\eta^{3} v_{r}}{r}\right)_{, r}\right|^{2}\right) d x\right)^{\frac{1}{2}}\left\|\nabla\left(\eta^{3} \phi\right)\right\|_{2, \mathcal{C}} \\
& \leq \frac{c C_{1}^{2}}{\ln ^{4}\left(e /\left|r_{1}\right|\right)}\left(\int_{\mathbb{C}} \frac{1}{r^{2} \ln ^{2}\left(e /\left|x^{\prime}\right|\right)}\left(\left|\left(\frac{\eta^{3} v_{r}}{r}\right)_{, 3}\right|^{2}+\left|\left(\frac{\eta^{3} v_{r}}{r}\right)_{, r}\right|^{2}\right) d x\right)^{\frac{1}{2}}\left\|\nabla\left(\eta^{3} \phi\right)\right\|_{2, \mathcal{C}}+C\left(v, \eta, r_{1}\right) .
\end{aligned}
$$

Here, it has been used boundedness of $\nabla^{2} v$ and $\nabla^{2} \omega$ in domain $\left.\left\{r>r_{1}\right\} \times\right]-1,0[$

Now, we again apply Lemma 2.2 and find another bound:

$$
\begin{aligned}
A_{0} \leq & \frac{c C_{1}^{2}}{\ln ^{4}\left(e /\left|r_{1}\right|\right)}\left(\left\|\nabla_{x^{\prime}}\left(\frac{\eta^{3} v_{r}}{r}\right)_{, 3}\right\|_{2, \mathcal{C}}^{2}\right. \\
& \left.+\left\|\nabla_{x^{\prime}}\left(\frac{\eta^{3} v_{r}}{r}\right)_{, r}\right\|_{2, \mathcal{C}}^{2}\right)^{\frac{1}{2}}\left\|\nabla\left(\eta^{3} \phi\right)\right\|_{2, \mathcal{C}}+C\left(v, \eta, r_{1}\right) .
\end{aligned}
$$


It remains to take into account the statement of Lemma 2.1 and conclude

$$
\begin{aligned}
A_{0} \leq & \frac{c C_{1}^{2}}{\ln ^{4}\left(e /\left|r_{1}\right|\right)}\left(\left\|\eta^{3} \Gamma_{, 3}\right\|_{2, \mathcal{C}}+C(v, \eta)\right)\left(\left\|\eta^{3} \nabla \Phi\right\|_{2, \mathcal{C}}+C(v, \eta)\right)+C\left(v, \eta, r_{1}\right) \\
\leq & \frac{c C_{1}^{2}}{\ln ^{4}\left(e /\left|r_{1}\right|\right)}\left\|\eta^{3} \nabla \Gamma\right\|_{2, \mathcal{C}}\left\|\eta^{3} \nabla \Phi\right\|_{2, \mathcal{C}} \\
& +C\left(v, \eta, r_{1}\right)\left(\left\|\eta^{3} \nabla \Gamma\right\|_{2, \mathcal{C}}+\left\|\eta^{3} \nabla \Phi\right\|_{2, \mathcal{C}}\right)+C\left(v, \eta, r_{1}\right) .
\end{aligned}
$$

Our next aim is a bound for $A_{32}$. Obviously, we have

$$
A_{32}=\int_{\mathcal{C}}\left(v_{\theta}\left(\frac{\eta^{3} v_{r}}{r}\right)_{, 3} \Phi\left(\eta^{3}\right)_{, r}-v_{\theta}\left(\frac{\eta v_{r}}{r}\right)_{, r} \Phi\left(\eta^{3}\right)_{, 3}\right) d x
$$

Here, we would like to use again that $v, \nabla v$, and $\nabla^{2} v$ are bounded over the support of $\nabla \eta$. In addition, we know that $\left|v_{\theta}\right| \leq|v|,|\Phi| \leq \nabla \omega,\left|\left(v_{r} / r\right)_{, 3}\right| \leq\left|\nabla^{2} v\right|$, and $\left|\left(v_{r} / r\right)_{, 3}\right| \leq\left|\nabla^{2} v\right|$. Therefore, we find

$$
A_{32} \leq C(v, \eta) \text {. }
$$

So, finally,

$$
\begin{aligned}
A_{3} \leq & \frac{c C_{1}^{2}}{\ln ^{4}\left(e /\left|r_{1}\right|\right)}\left\|\eta^{3} \nabla \Gamma\right\|_{2, \mathcal{C}}\left\|\eta^{3} \nabla \Phi\right\|_{2, \mathcal{C}} \\
& +C\left(v, \eta, r_{1}\right)\left(\left\|\eta^{3} \nabla \Gamma\right\|_{2, \mathcal{C}}+\left\|\eta^{3} \nabla \Phi\right\|_{2, \mathcal{C}}\right)+C\left(v, \eta, r_{1}\right) .
\end{aligned}
$$

Combing all the estimates made on this step, we shall have:

$$
\begin{aligned}
& \left.\frac{1}{2} \partial_{t} \int_{\mathcal{C}}\left(\Phi \eta^{3}\right)^{2}+\left(\Gamma \eta^{3}\right)^{2} d x+\int_{\mathcal{C}}\left(\eta^{3}|\nabla \Phi|\right)^{2}+\eta^{3}|\nabla \Gamma|\right)^{2} d x \\
& \leq\left(\frac{c C_{1}}{\ln \left(e / r_{1}\right)}+\frac{c C_{1}^{2}}{\ln ^{4}\left(e /\left|r_{1}\right|\right)}\right)\left\|\eta^{3} \nabla \Gamma\right\|_{2, \mathcal{C}}\left\|\eta^{3} \nabla \Phi\right\|_{2, \mathcal{C}} \\
& \quad+C\left(v, \eta, r_{1}\right)\left(\left\|\eta^{3} \nabla \Gamma\right\|_{2, \mathcal{C}}+\left\|\eta^{3} \nabla \Phi\right\|_{2, \mathcal{C}}\right)+C\left(v, \eta, r_{1}\right) .
\end{aligned}
$$

Here, it is assumed that a number $\left.r_{1} \in\right] 0,1 / 4[$ so small as

$$
\frac{c C_{1}}{\ln \left(e / r_{1}\right)}+\frac{c C_{1}^{2}}{\ln ^{4}\left(e /\left|r_{1}\right|\right)}<2
$$

So, if the latter condition holds, the key estimate can be derived by more or less standard arguments. It is as follows:

$$
\sup _{-1<t<0} \int_{\mathcal{C}} \eta^{6}\left(|\Gamma|^{2}+|\Phi|^{2}\right) d x+\int_{Q}\left(\eta^{3}|\nabla \Phi|\right)^{2}+\left(\eta^{3}|\nabla \Gamma|\right)^{2} d x d t \leq C\left(v, \eta, r_{1}\right) .
$$

Step 4. Final Conclusion. Now, let us show that the origin $z=0$ is a regular point of $v$. To this end, we are going to use the estimate proved at the previous step. It can be re-written to the form:

$$
\left|\eta^{3} \Phi\right|_{2, Q}^{2}+\left|\eta^{3} \Gamma\right|_{2, Q}^{2}<\infty
$$

where as usual $|f|_{2, Q}^{2}=\sup _{-1<t<0}\|f(\cdot, t)\|_{2, \mathcal{C}}^{2}+\|\nabla f\|_{2, Q}^{2}$. Since $\omega_{\theta}=r \Gamma$, one can state that $\left|\eta^{3} \omega_{\theta}\right|_{2, Q}<\infty$ as well. It will be used to make various estimates of the solution to equation (2.3). Indeed, the elliptic theory implies two classical bounds:

$$
\left\|\nabla\left(\eta^{3} \bar{v}\right)\right\|_{2, \mathcal{C}} \leq c\left\|\omega_{\theta} \eta^{3}\right\|_{2, \mathcal{C}}+c\left\|\left|\nabla \eta^{3}\|\bar{v} \mid\|_{2, \mathcal{C}}\right.\right.
$$

and

$$
\left\|\nabla^{2}\left(\eta^{3} \bar{v}\right)\right\|_{2, \mathcal{C}} \leq c\left|\left\|\nabla \eta^{3}\right\| \nabla \bar{v}\right|\left\|_{2, \mathcal{C}}+c\left|\left\|\nabla^{2} \eta^{3}\right\| \bar{v}\right|\right\|_{2, \mathcal{C}}+c\left\|\operatorname{curl}\left(\omega_{\theta} \eta^{3} e_{\theta}\right)\right\|_{2, \mathcal{C}} .
$$


Let us notice that

$$
\operatorname{curl}\left(\omega_{\theta} \eta^{3} e_{\theta}\right)=-\left(\omega_{\theta} \eta^{3}\right)_{, 3} e_{r}+\left(\left(\omega_{\theta} \eta^{3}\right)_{, r}+\Gamma \eta^{3}\right) e_{3}
$$

and

$$
|\nabla \bar{v}| \leq|\nabla v|
$$

Then, our previous arguments can be exploited to describe properties of the solution $v$ in the support of $\nabla \eta$ and conclude that the quantity $\left|\nabla\left(\eta^{3} \bar{v}\right)\right|_{2, Q}$ is bounded that in turn yields boundedness of two norms: $\left\|\nabla\left(\eta^{3} \bar{v}\right)\right\|_{\frac{10}{3}, Q}$ and $\left\|\nabla\left(\eta^{3} \bar{v}\right)\right\|_{\infty, \frac{10}{3}, Q}$. So, for all sufficiently small $R$, we find

$$
\frac{1}{R^{2}} \int_{Q(R)}|\bar{v}|^{3} d z \leq c R^{\frac{11}{5}}\left\|\eta^{3} \bar{v}\right\|_{\infty, \frac{10}{3}, Q}^{3} \rightarrow 0
$$

as $R \rightarrow 0$.

It remains to understand what happens with $v_{\theta}$. Indeed, we have

$$
\eta^{3} v_{\theta}\left(r, x_{3}, t\right)=\int_{-1}^{x_{3}}\left(\eta^{3} v_{\theta}\right)_{, 3}(r, y, t) d y
$$

and thus

$$
\sup _{-1 \mid<x_{3}<1}\left|\eta^{3} v_{\theta}\left(r, x_{3}, t\right)\right| \leq c\left(\left.\int_{-1}^{1}\left|\left(\eta^{3} v_{\theta}\right)_{, 3}\right|(r, y, t)\right|^{\frac{10}{3}} d y\right)^{\frac{3}{10}} .
$$

The latter inequality can be re-written so that

$$
\sup _{-1 \mid<x_{3}<1} \frac{1}{r}\left|\eta^{3} v_{\theta}\left(r, x_{3}, t\right)\right| \leq c\left(\int_{-1}^{1}\left(\left|\Phi \eta^{3}\right|+\left|\left(\eta^{3}\right)_{, 3}\right| v_{\theta} \mid / r\right)^{\frac{10}{3}}(r, y, t) d y\right)^{\frac{3}{10}} .
$$

Taking into account the inequality $\left|v_{\theta}\right| / r \leq|\nabla v|$ and boundedness of $|\nabla v|$ in the support of $\nabla \eta$, after integration by parts, we get:

$$
\int_{-1}^{0} \int_{0}^{1}\left(\sup _{-1 \mid<x_{3}<1} \frac{1}{r}\left|\eta^{3} v_{\theta}\left(r, x_{3}, t\right)\right|\right)^{\frac{10}{3}} r d r d t \leq c\left\|\Phi \eta^{3}\right\|_{\frac{10}{3}, Q}^{\frac{10}{3}}+C(v, \eta)<\infty .
$$

So, for sufficiently small $R>0$, we have

$$
\begin{aligned}
& \frac{1}{R^{2}} \int_{Q(R)}\left|v_{\theta}\right|^{3} d z \leq c \frac{1}{R^{\frac{3}{2}}}\left[\int_{Q(R)}\left|v_{\theta}\right|^{\frac{10}{3}} d z\right]^{\frac{9}{10}} \\
& \leq c \frac{1}{R^{\frac{3}{2}}}\left[\int_{-R^{2}}^{0} \int_{0}^{R}\left(R^{\frac{10}{3}} \int_{-R}^{R}\left|v_{\theta} / r\right|^{\frac{10}{3}} d x_{3}\right) r d r d t\right]^{\frac{9}{10}} \\
& \leq c \frac{R^{\left(\frac{10}{3}+1\right) \frac{9}{10}}}{R^{\frac{3}{2}}}\left[\int_{-1}^{0} \int_{0}^{1} \sup _{-1<x_{3}<1}\left|\frac{v_{\theta} \eta^{3}\left(r, x_{3}, t\right)}{r}\right|^{\frac{10}{3}} r d r d t\right]^{\frac{9}{10}} \rightarrow 0
\end{aligned}
$$

as $R \rightarrow 0$. According to the partial regularity theory for the Navier-Stokes equations and to (2.5), the origin $z=0$ is a regular point of $v$. Theorem 1.2 is proved. 


\section{Proof of Theorem 1.4}

Here, we are going to use two scale-invariant inequalities proved in [20]. They are as follows:

$$
C(\varrho) \leq c \varepsilon \mathcal{E}(\varrho)+c_{1}(s, l, \varepsilon) G(\varrho), \quad G(\varrho)=\left(M^{s, l}(\varrho)\right)^{\frac{1}{l(2-3 / s-2 / l)}}
$$

and

$$
\mathcal{E}(\theta \varrho) \leq\left(\theta \mathcal{E}(\varrho)+\frac{1}{\theta^{2}} C(\varrho)+\frac{1}{\theta^{\frac{4}{3}}} C^{\frac{2}{3}}(\varrho)\right)
$$

where $\varepsilon>0,0<\theta<1$ and $0<\varrho<1$, various scale-invariant quantities are defined as

$$
\begin{aligned}
& \mathcal{E}(\varrho)=E(\varrho)+A(\varrho)+D(\varrho), \quad C(\varrho)=\frac{1}{\varrho^{2}} \int_{Q(\varrho)}|v|^{3} d z, \\
& E(\varrho)=\frac{1}{\varrho} \int_{Q(\varrho)}|\nabla v|^{2} d z, \quad A(\varrho)=\frac{1}{\varrho} \sup _{-\varrho^{2}<t<0} \int_{\mathcal{C}(\varrho)}|v(x, t)|^{2} d x, \\
& D(\varrho)=\frac{1}{\varrho^{2}} \int_{Q(\varrho)}|q|^{\frac{3}{2}} d z, \quad M^{s, l}(\varrho)=\frac{1}{\varrho^{\kappa}} \int_{-\varrho^{2}}^{0} d t\left(\int_{\mathcal{C}(\varrho)}|v|^{s} d x\right)^{\frac{l}{s}},
\end{aligned}
$$

$\kappa=l\left(\frac{3}{s}+\frac{2}{l}-1\right)$ and numbers $s$ and $l$ satisfies restrictions:

$$
\frac{1}{2}>\frac{3}{s}+\frac{2}{l}-\frac{3}{2}>\max \left\{\frac{1}{2 l}, \frac{1}{2}-\frac{1}{l}\right\} .
$$

From the above inequalities, it can be easily derived

$$
\mathcal{E}(\theta \varrho) \leq c\left[\left(\theta+\frac{\varepsilon}{\theta^{2}}+\frac{\varepsilon^{\frac{1}{2}}}{\theta}\right) \mathcal{E}(\varepsilon)+c_{2}(\varepsilon, \theta)+c_{3}(s, l, \varepsilon, \theta) G(\varepsilon)\right] .
$$

Next, we choose a positive number $\theta$ to satisfy the inequality $c \theta \leq 1 / 4$ and then pick up a positive number $\varepsilon$ so that

$$
\frac{\varepsilon}{\theta^{2}}+\frac{\varepsilon^{\frac{1}{2}}}{\theta}<\frac{1}{4}
$$

Hence, we have

$$
\mathcal{E}(\theta \varrho) \leq \frac{1}{2} \mathcal{E}(\varrho)+c+c_{5}(s, l) G(\varrho)
$$

After iterations, we get the inequality

$$
\mathcal{E}\left(\theta^{k} \varrho\right) \leq \frac{1}{2^{k}} \mathcal{E}(\varrho)+c+c_{5}(s, l) \sum_{i=0}^{k-1} \frac{G\left(\theta^{i} \varrho\right)}{2^{k-1-i}}
$$

being valid for each natural $k$.

Now, assume that condition (1.7) holds, set $s=\frac{7}{4}$ and $l=10$, and try to evaluate the quantity $M^{s, l}$ and thus the quantity $G$. So, we have

$$
G(\varrho) \leq \frac{1}{\varrho^{\frac{64}{7}}} \int_{-\varrho^{2}}^{0} d t\left(2 \pi_{0} \int_{-\varrho}^{\varrho} d x_{3} \int_{0}^{\varrho}\left(\frac{c}{r} \ln ^{\alpha} \ln \frac{1}{r}\right)^{\frac{7}{4}} r d r\right)^{\frac{40}{7}}
$$

To estimate the above integral, we assume that a number $\alpha$ and the variable $\varrho$ are positive and sufficiently small. Then, integration by parts gives us:

$$
\int_{0}^{\varrho}\left(\frac{1}{r} \ln ^{\alpha} \ln \frac{1}{r}\right)^{\frac{7}{4}} r d r=4 \varrho^{\frac{1}{4}} \ln ^{\frac{7 \alpha}{4}} \ln \frac{1}{\varrho}+7 \alpha \int_{0}^{\varrho} \frac{1}{\varrho^{\frac{3}{4}}} \frac{1}{\ln ^{1-\frac{7}{4} \alpha} \ln \frac{1}{\varrho}} \frac{1}{\ln \frac{1}{\varrho}} d r \leq c \varrho^{\frac{1}{4}} \ln \frac{7 \alpha}{4} \ln \frac{1}{\varrho} .
$$


As a result,

$$
G(\varrho) \leq c \ln ^{\frac{35}{3} \alpha} \ln \frac{1}{\varrho}
$$

Therefore,

$$
\mathcal{E}\left(\theta^{k} \varrho\right) \leq \frac{1}{2^{k}} \mathcal{E}(\varrho)+c+c \ln ^{\frac{35}{3} \alpha} \ln \frac{1}{\theta^{k} \varrho} .
$$

From the last estimate, it follows that

$$
\mathcal{E}(\varrho) \leq C(\mathcal{E}(1 / 2), \alpha) \ln ^{\frac{35}{3} \alpha} \ln 1 / \varrho
$$

for all $0<\varrho \leq 1 / 2$. It remains to notice $f(R)+M(R) \leq c \sqrt{\mathcal{E}(R)}$. Theorem 1.4 has been proved.

\section{Corrections to the Preprint [18]}

In the note [18], there is an error in calculations of a certain integral at the very end of Proposition 1.4. It can be corrected so that all results of note [18] remain true. The correct version of Proposition 1.4 of [18] is Proposition 1.3 of the present note. Keeping notation of the note [18], let us comment to changes to the proof.

The first comment is related to more delicate estimate of $\hat{\beta}_{2}$ and is as follows.

Obviously, there exists a number $0<R_{* 5}\left(M_{0}, c_{*}, \alpha\right) \leq \min \left\{1 / 6, R_{* 2}\right\}$ such that

$$
2 c\left(M_{0}, c_{*}\right) \ln ^{224 \alpha-1} \sqrt{\ln \frac{1}{R}} \leq \frac{1}{2}
$$

and

$$
c\left(M_{0}, c_{*}\right) \ln ^{224 \alpha} \sqrt{\ln \frac{1}{R}} \geq \ln g(2 R)
$$

for $0<R \leq R_{* 5}\left(M_{0}, c_{*}, \alpha\right)$ and thus

$$
\begin{aligned}
& -c\left(M_{0}, c_{*}\right) \ln ^{224 \alpha} \sqrt{\ln \frac{1}{R}} \\
& =-2 c\left(M_{0}, c_{*}\right) \ln ^{224 \alpha} \sqrt{\ln \frac{1}{R}}++c\left(M_{0}, c_{*}\right) \ln ^{224 \alpha} \sqrt{\ln \frac{1}{R}} \geq \\
& -\left(2 c\left(M_{0}, c_{*}\right) \ln ^{224 \alpha-1} \sqrt{\ln \frac{1}{R}}\right) \ln \sqrt{\ln \frac{1}{R}}+\ln g(2 R) \\
& \geq-\ln \left(\ln \frac{1}{R}\right)^{\frac{1}{4}}+\ln g(2 R) .
\end{aligned}
$$

Now, the number $\hat{\beta}_{2}$ is estimated as follows:

$$
\hat{\beta}_{2} \geq\left(\ln \frac{1}{R}\right)^{-\frac{1}{4}} g(2 R)
$$

for $0<R \leq R_{* 5}\left(M_{0}, c_{*}, \alpha\right)$. Then

$$
\beta(2 R)=\frac{c}{\ln ^{\frac{3}{4}}(1 / R)}
$$


and estimate of $\eta_{k}$ is as follows:

$$
\begin{aligned}
\leq & -c \sum_{i=0}^{k}\left(\ln \left(2^{2 i-1} / R\right)\right)^{-\frac{3}{4}}=-c \sum_{i=0}^{k} \frac{1}{(i \ln 4+\ln (1 /(2 R)))^{\frac{3}{4}}} \\
& \leq-c \int_{0}^{k+1} \frac{d x}{(x \ln 4+\ln (1 /(2 R)))^{\frac{3}{4}}}= \\
& =-\left.\frac{4 c}{\ln 4}(x \ln 4+\ln (1 /(2 R)))^{\frac{1}{4}}\right|_{0} ^{k+1} \\
& \left.=-c\left(\ln ^{\frac{1}{4}}\left(2^{2 k+1} / R\right)-\ln ^{\frac{1}{4}}(1 /(2 R))\right)\right) .
\end{aligned}
$$

So, (1.5) follows.

Changes in the proof of Theorem 1.3 in [18] are based on the inequality

$$
\left|\sigma\left(\varrho, x_{3}, t\right)\right| \leq C\left(c_{*}, \alpha\right) e^{-c \ln \frac{1}{4}(1 /(2 \varrho))} \Sigma_{0} \leq C\left(c_{*}, \alpha\right) \Sigma_{0} \frac{m !}{c^{m} \ln ^{\frac{m}{4}}(1 /(2 \varrho))},
$$

being valid for all natural numbers $m$, and references to [24], [9], and [11].

More cases of slightly growing scale-invariant quantities are considered in the preprint of $\mathrm{H}$. Chen, T.-P. Tsai, and T. Zhang "Remarks on local regularity of axisymmetric solutions to the 3D Navier-Stokes equations", arXiv:2201.01766v1.

Acknowledgements. The work is supported by the Grant No. RFBR 20-01-00397.

Data availability statement Data sharing not applicable to this article as no datasets were generated or analysed during the current study.

\section{Declarations}

Conflict of interest The authors declares that they have no competing interests.

Open Access. This article is licensed under a Creative Commons Attribution 4.0 International License, which permits use, sharing, adaptation, distribution and reproduction in any medium or format, as long as you give appropriate credit to the original author(s) and the source, provide a link to the Creative Commons licence, and indicate if changes were made. The images or other third party material in this article are included in the article's Creative Commons licence, unless indicated otherwise in a credit line to the material. If material is not included in the article's Creative Commons licence and your intended use is not permitted by statutory regulation or exceeds the permitted use, you will need to obtain permission directly from the copyright holder. To view a copy of this licence, visit http://creativecommons.org/licenses/by/4.0/.

Publisher's Note Springer Nature remains neutral with regard to jurisdictional claims in published maps and institutional affiliations.

\section{References}

[1] Caffarelli, L., Kohn, R.-V., Nirenberg, L.: Partial regularity of suitable weak solutions of the Navier-Stokes equations. Comm. Pure Appl. Math. XXXV, 771-831 (1982)

[2] Chae, D., Lee, J.: On the regularity of the axisymmetric solutions of the Navier-Stokes equations. Math. Z. 239, 645-671 (2002)

[3] Chen, C., Fang, D., Zhang, T.: Regularity of 3D axisymmetric Navier-Stokes equations. Discr. Contin. Dyn. Sys. - A 37(4), 1923-1939 (2017)

[4] Chen, C., Strain, R.M., Yau, H., Tsai, T.: Lower bounds on the blow-up rate of the axisymmetric Navier-Stokes equations II. Comm. Part. Diff. Equa. 34, 203-232 (2009)

[5] Hmidi, T., Rousset, F.: Global well-posedness for the Euler-Boussinesq system with axisymmetric data. J. Funct. Anal. 260, 745-796 (2011)

[6] Ladyzhenskaya, O.A.: On unique solvability of the three-dimensional Cauchy problem for the Navier-Stokes equations under the axial symmetry. Zap. Nauchn. Sem. LOMI 7, 155-177 (1968) 
[7] Ladyzhenskaya, O.A., Seregin, G.A.: On partial regularity of suitable weak solutions to the three-dimensional NavierStokes equations. J. math. fluid mech. 1, 356-387 (1999)

[8] Lei, Z., Zhang, Q.: A Liouville theorem for the axially symmetric Navier-Stokes equations. J. Funct. Anal. 261, 2323-2345 (2011)

[9] Lei, Z., Zhang, Q.: Criticality of the axially symmetric Navier-Stokes equations. Pacif. J. Math. 289(1), 169-187 (2017)

[10] Leonardi, S., Malek, J., Necas, J., Pokorny, M.: On axially simmetric flows in $\mathbb{R}^{3}$. ZAA 18, 639-649 (1999)

[11] Li, Z., Pan, X.: Some remarks on regularity criteria of axially symmetric Navier-Stokes equations. Commun. Pure Appl. Anal. 18(3), 1333-1350 (2019)

[12] Lin, F.-H.: A new proof of the Caffarelli-Kohn-Nirenberg theorem. Comm. Pure Appl. 51, 241-257 (1998)

[13] Miao, C., Zheng, X.: On the global well-posedness for the Boussinesq system with horizontal dissipation. Comm. Math. Phys. 321(1), 33-67 (2013)

[14] Neustupa, J., Pokorny, M.: Axisymmetric flow of Navier-Stokes fluid in the whole space with non-zero angular velocity compnents. Math. Bohemica 126, 469-481 (2001)

[15] Pan, X.: Regularity of solutions to axisymmetric Navier-Stokes equations with a slightly supercritical condition. J. Different. Equ. 260(12), 8485-8529 (2016)

[16] Pokorny, M.: A regularity criterion for the angular velocity component in the case of axisymmetric Navier-Stokes equations (2001)

[17] Seregin, G.: Local regularity of axisymmetric solutions to the Navier-Stokes equations. Anal. Math. Phys. 10(4), 46-20 (2020)

[18] Seregin, G.: A slightly supercritical condition of regularity of axisymmetric solutions to the Navier-Stokes equations arXiv:2109.09344v1

[19] Seregin, G., Sverak, V.: On Type I singularities of the local axi-symmetric solutions of the Navier-Stokes equations. Communicat. PDE's 34, 171-201 (2009)

[20] Seregin, G., Sverak, V.: Regularity criteria for Navier-Stokes solutions. Handbook of mathematical analysis in mechanics of viscous fluids, 829-867, Springer, Cham

[21] Seregin, G., Zajaczkowski, W.: A sufficient condition of regularity for axially symmetric solutions to the Navier-Stokes equations. SIAM J. Math. Anal 39, 669-685 (2007)

[22] Seregin, G., Zhou, D.: Regularity of solutions to the Navier-Stokes equations in $\dot{B}_{\infty, \infty}^{-1}$. Zapiski POMI 407, 119-128 (2018)

[23] Ukhovskij, M.R., Yudovich, V.L.: Axially symmetric motions of ideal and viscous fluids filling all space. Prikl. Mat. Mech. 32, 59-69 (1968)

[24] Wei, D.: Regularity criterion to the axially symmetric Navier-Stokes equations. J. Math. Anal. Appl. 435(1), 402-413 (2016)

[25] Zhang, P., Zhang, T.: Global axisymmetric solutions to the three-dimensional Navier-Stokes equations system. Int. Math. Res. Not. 2014, 610-642 (2014)

G. Seregin

University of Oxford, Mathematical Institute,

OxPDE

Oxford

UK

and
St Petersburg Department of Steklov Mathematical

Institute, RAS

St Petersburg

Russia

e-mail: seregin@maths.ox.ac.uk

(accepted: January 19, 2022; published online: February 19, 2022) 